\title{
Web Data Cleansing for Information Retrieval using Key Resource Page Selection
}

\author{
Yiqun Liu, Canhui Wang \\ State Key Lab of Intelligent technology \& systems \\ Tsinghua University \\ Beijing, China P.R. \\ Liuyiqun03@mails.tsinghua.edu.cn
}

\author{
Min Zhang, Shaoping Ma \\ State Key Lab of Intelligent technology \& systems \\ Tsinghua University \\ Beijing, China P.R. \\ \{z-m, msp\}@tsinghua.edu.cn
}

\begin{abstract}
With the page explosion of WWW, how to cover more useful information with limited storage and computation resources becomes more and more important in web IR research. Using web page non-content feature analysis, we proposed a clustering-based method to select high quality pages from the whole page set. Although the result page set contains only $44.3 \%$ of the whole collection, it is related with more than $98 \%$ of links and covers about $90 \%$ of key information. Link property and retrieval affects are also observed and experiment results show that key resource selection method is more suitable for the job of data cleansing and the result page set outperforms the whole collection by smaller size and better retrieval performance.
\end{abstract}

\section{Categories and Subject Descriptors}

H.3.3 [Information Storage and Retrieval]: Information Search and Retrieval

\section{General Terms \\ Experimentation}

\section{Keywords}

Web data cleansing, Non-content feature, Web IR.

\section{INTRODUCTION}

The explosive growth of data on the Web makes information management and knowledge discovery increasingly difficult. In one hand, not all pages can be collected by web information management tools. In the other hand, not all pages collected are useful and not all information on these pages is high-qualified, since the web is filled with noisy, unreliable, low-quality and sometimes contradictory data. It would be extremely helpful for web search engines to identify the quality of web pages independent of a given user request, so that they can index more high-quality pages with limited resources. This is called one of web search engine's challenges. Link-based approaches such as PageRank [3] and HITS [3] can partly solve the problem. However, they only use link structures of the web and a better estimate should require additional non-content sources of information both within a page and across different pages.

In this paper we proposed a web data cleansing method. Noncontent features including but not limited to link analysis features are involved in this method. Clustering-based algorithms are adopted to select a certain kind of high quality pages called key resources. Main contributions of our work are:

Copyright is held by the International World Wide Web Conference Committee (IW3C2). Distribution of these papers is limited to classroom use, and personal use by others.

$W W W$ 2005, May 10-14, 2005, Chiba, Japan.

ACM 1-59593-051-5/05/0005.
[1] A non-content feature study is conducted to draw a clear picture of the differences between high quality pages and ordinary web pages. [2] A cluster-based method is proposed to automatically select important web pages according to whether they have chance to be key resources. This method makes use of both prior knowledge and page's non-content features. [3] The possibility of achieving better retrieval performance with a preselected page set is discussed.

\section{NON-CONTENT FEATURES OF KEY RESOURCE PAGES}

Key resource page is a kind of high quality web page which provides as much credible information as possible on a certain topic [2]. It is different from ordinary pages even if they are relevant to the same topic. From relevant but not key resource pages one only gets information covering a small part of knowledge and can't read more. However, key resource page either provides credible information, or offers plenty of useful information that can be obtained via only one click.

Feature selection is the key point in the procedure of separating key resource pages. Data preparation should be a queryindependent process for web search engines so only non-content features can be adopted, which are shown in Table 1.

Table 1 Differences in non-content feature average value between ordinary pages and key resource pages ${ }^{2}$

\begin{tabular}{|c|c|c|}
\hline Average Value & .GOV & Key Resource \\
\hline In-degree & 9.94 & 153.12 \\
\hline URL length (in 4 categories) & 3.85 & 3.07 \\
\hline In-site out-link anchor text rate & 0.06 & 0.12 \\
\hline In-site out-link number & 17.58 & 37.70 \\
\hline Document Length (in words) & 7037.43 & 9008.02 \\
\hline
\end{tabular}

In-site out-link is defined as an out-link navigating to another page located in the same site. This kind of link is specially treated because key resource pages should have enough in-site out-link to connect to other pages in the same site and enough in-site out-link anchor text to give a brief view of these pages.

There are differences in non-content feature distribution between ordinary and key resource pages. It means that these features can tell the two kinds of pages from each other.

${ }^{1}$ Our work is supported by the Chinese National Key Foundation Research \& Development Plan (2004CB318108) and Natural Science Foundation (60223004, 60321002, 60303005)

2 .GOV (ordinary page set) is a crawl of $1.25 \mathrm{M}$ Web pages from gov domain composed of over $18 \mathrm{G}$ data. Training set is composed of relevant answers of TREC 2002's web track. 


\section{A CLUSTER-BASED KEY RESOURCE SELECTION ALGORITHM}

Although we can get several positive examples for key resource pages, there are too many reasons for one page to be a non key resource page. Negative instance collecting will be very difficult or even impossible. That is why supervised classification analysis can't be performed for key resource selection. A clustering-based algorithm is therefore used to combine features to do this task. Figure 1 show how the algorithm performs on .GOV corpus.

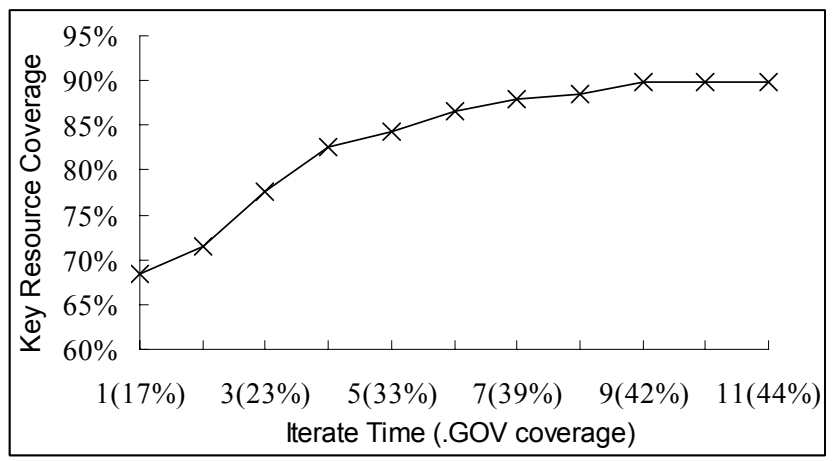

Figure 1. Key Resource Coverage varies with iterate times.

At first, .GOV coverage is assigned an initial value (1/6) to calculate the negative instance set centroid. Key resource test set is composed of relevant answers of TREC 2003's topic distillation task (516 pages related with 50 topics). From Figure 1 we can see that it is possible to cover $90 \%$ test set pages with about $44 \%$ of all pages.

\section{EXPERIMENTS AND DISCUSSIONS}

\subsection{Link Analysis Experiment Results}

Page set developed with our key resource selection method covers a majority of links in the whole collection. There are altogether 1247753 pages and 10185630 hyperlinks in .GOV corpus and this page set are related with over $98 \%$ links. It means although more pages are outside the page set in .GOV, there would be almost no hyperlinks if pages inside the set were taken. This hyperlink distribution shows that key resource pages would be the top scored ones in link structure analysis.

\subsection{Retrieval Experiment Results}

According to the query log analysis of Alta Visa by Broder in [1], web search engine queries are divided into 3 categories, which are Navigational, Informational and Transactional. In order to simulate web search user activity as close as possible, we build a query set in which each type of query has the same coverage as in query $\log$ analysis. Details are shown in Table 2.

Table 2 Query type distribution in our test query set

\begin{tabular}{|c|c|c|}
\hline Type of query & Query log analysis in [1] & Test query set \\
\hline Navagational & $20 \%$ & $10(20 \%)$ \\
\hline Informational & $50 \%$ & \multirow{2}{*}{$40(80 \%)$} \\
\hline Transactional & $30 \%$ & \\
\hline
\end{tabular}

We randomly selected 10 topics from TREC 2003's known item search task and 40 topics from its topic distillation task to build this query set. Corresponding relevant answers are used to judge the effectiveness of retrieval experiment results.
Corresponding retrieval Experiment results are shown in Figure 2 and Table 3. BM25 weighting in full text index and default parameter tuning are applied in both page sets.

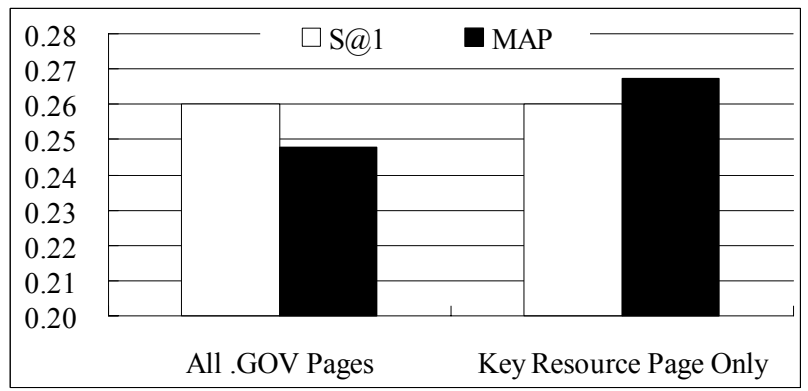

Figure 2 Retrieval performances in the measure of success rate at $1^{\text {st }}$ document and mean average precision.

Table 3 Retrieval performance for different type of queries

\begin{tabular}{|c|c|c|}
\hline Page Set & $\begin{array}{c}\text { Informational } \\
\text { queries (P } \mathbf{a} \mathbf{1 0})\end{array}$ & $\begin{array}{c}\text { Navigational queries } \\
\text { (MRR) }\end{array}$ \\
\hline .GOV & 0.1025 & 0.7443 \\
\hline Key resource & 0.1275 & 0.7278 \\
\hline
\end{tabular}

From these experiment results, we can get the following conclusions: First, web page cleansing via selecting key resources is useful because the page set after cleansing outperforms entire page set by smaller size and better retrieval performance. It is composed of less than half of GOV pages but get better overall performance in the measure of MAP. Second, the entire page set gets higher MRR for navigational type queries according to Table 3 because it is unavoidable to reduce part of useful information in the process of data cleansing. However, navigational queries only cover a small part $(20 \%)$ of web search request and overall performance improves with the data cleansing method.

\section{CONCLUSIONS AND FUTURE WORK}

In this paper, a key resource selection approach based on $\mathrm{K}$ means clustering for web data cleansing is proposed. The whole collection, which is $19 \mathrm{G}$.GOV web data set, is divided into two sets. By doing so, only $44.3 \%$ pages are selected as key resources, which will be kept for indexing and retrieval by IR systems. This set contains $98 \%$ links of the whole collection. Retrieval experiments get better overall performance although this page set has much fewer pages than the whole collection.

Future study will focus on following aspects: Is it possible to identify navigational search destiny pages query-independently so that we can improve performance for this kind of queries? Can we rank pages instead of filter them with a similar non-content feature analysis process for web data cleansing? Is there a best trade off between page set size and retrieval performance?

\section{REFERENCES}

[1] A. Broder, A taxonomy of web search. SIGIR Forum Volume 36 Number 2, 2002.

[2] D. Hawking and N. Craswell. Overview of the TREC-2003 Web track. NIST Special Publication: SP 500-255, The Twelfth Text Retrieval Conference (TREC 2003), 2003.

[3] J. Kleinberg. Authoritative sources in a hyperlinked environment. Journal of the ACM, Volume 46 (5), 1999.

[4] S. Brin and L. Page. The anatomy of a large-scale hypertextual web search engine. Proceedings of the 7 th World-Wide Web Conference (WWW7), 1998. 